\title{
Dietary factors, gut microbiota, and serum trimethylamine-N-oxide associated with cardiovascular disease
}

\section{Zhendong Mei}

Fudan University https://orcid.org/0000-0003-1129-3147

\section{Guo-Chong Chen}

Yeshiva University Albert Einstein College of Medicine

\section{Zheng Wang}

Yeshiva University Albert Einstein College of Medicine

Mykhaylo Usyk

Yeshiva University Albert Einstein College of Medicine

\section{Bing Yu}

University of Texas Health Science Center at Houston

\section{Yoshiki Vazquez Baeza}

University of California San Diego

\section{Greg Humphrey}

University of California San Diego

\section{Rodolfo Salido Benitez}

University of California San Diego

Jun Li

Harvard University T H Chan School of Public Health Jessica S. Williams-Nguyen

Fred Hutchinson Cancer Research Center

\section{Martha L. Daviglus}

University of Illinois at Chicago College of Medicine

\section{Lifang Hou}

Northwestern University

\section{Jianwen Cai}

University of North Carolina at Chapel Hill

\section{Yan Zheng}

Fudan University

\section{Rob Knight}

University of California San Diego

Robert D. Burk 
Yeshiva University Albert Einstein College of Medicine

\section{Eric Boerwinkle}

University of Texas Health Science Center at Houston

\section{Robert C. Kaplan}

Yeshiva University Albert Einstein College of Medicine

Qibin Qi ( $\nabla$ qibin.qi@einsteinmed.org )

Albert Einstein College of Medicine https://orcid.org/0000-0002-2687-1758

\section{Research}

Keywords: Cardiovascular disease, Diet, Gut microbiota, Trimethylamine-N-oxide

Posted Date: August 24th, 2020

DOl: https://doi.org/10.21203/rs.3.rs-61643/v1

License: (c) (i) This work is licensed under a Creative Commons Attribution 4.0 International License. Read Full License 


\section{Abstract \\ Background}

Trimethylamine-N-oxide (TMAO), a diet-derived and gut microbiota-related metabolite, is associated with cardiovascular disease (CVD). However, the major dietary determinants and the specific gut bacterial taxa related to TMAO remain to be identified in humans. We aimed to identify dietary and gut microbial factors independently and jointly associated with circulating TMAO.

\section{Results}

We examined usual dietary intake, fecal gut microbiome profiled by shotgun metagenomics, and their interactions in relation to serum TMAO and its precursors among up to 3972 adult participants from the Hispanic Community Health Study/Study of Latinos. We confirmed the positive association between TMAO and prevalent CVD $\left(\mathrm{OR}=1.28, P=4.36 \cdot 10^{-4}\right)$. Fish $\left(P=1.26 \cdot 10^{-17}\right)$, red meat $\left(P=3.33 \cdot 10^{-16}\right)$, and egg $\left(P=3.89 \cdot 10^{-5}\right)$ intakes were top dietary factors positively associated with serum TMAO independently of each other. Red meat and egg intakes, but not fish intake, were positively associated with serum TMAO precursors (e.g., carnitine, choline). We identified 9 gut bacterial species significantly associated with serum TMAO after multiple testing correction (FDR < 0.05). All 4 TMAO-positivelyassociated bacteria belong to the Clostridiales order, 3 of which may have homologous genes encoding carnitine monooxygenase, an enzyme converting carnitine to trimethylamine (TMA)/TMAO. The red meatTMAO association was more pronounced for participants with higher abundance of these 4 bacterial species than those with lower abundance $\left(P_{\text {interaction }}=0.013\right)$, but such microbial modification was not observed for fish-TMAO or egg-TMAO associations.

\section{Conclusion}

In US Hispanics/Latinos, fish, red meat, and egg intakes are major dietary factors associated with serum TMAO. The identified potential TMA-producing gut microbiota and microbial modification on the red meat-TMAO association support microbial TMA/TMAO production from dietary carnitine, while the fishTMAO association is independent of gut microbiota.

\section{Introduction}

Trimethylamine-N-oxide (TMAO), a potential diet-derived and gut microbiota (GMB)-dependent metabolite, has been associated with an increased risk of cardiovascular disease (CVD) independent of traditional risk factors in numerous population studies[1-3]. Environmental and host factors, such as GMB, dietary intake, and host genetics, are thought to independently and jointly influence circulating levels of TMAO[4], but data from human population studies are still limited. Identifying potential drivers for alternations in circulating TMAO may have preventive and therapeutic implications in CVD. 
Dietary trimethylamine (TMA)-containing nutrients, including choline, phosphatidylcholine, carnitine, and betaine abundant in animal foods such as red meat (e.g., beef and pork) and eggs [1, 5], can be converted to TMAO in humans through a series of physiological processes ${ }^{2}$. Specifically, TMA is liberated from TMA-containing nutrients by intestinal bacteria[4], passively absorbed into the circulation system and then oxidized to TMAO by flavin monooxygenases in the liver[1]. In addition to TMAO produced from dietary precursors, preformed TMAO in humans[6] and animals[7] can be absorbed in a manner not involving gut microbes. A recent human feeding study has shown that fish (cod fillet) contains a high concentration of TMAO; and that circulating TMAO was elevated within 15 minutes of fish consumption. [6] However, human population studies assessing the relationship between dietary factors and TMAO have been inconclusive,[8-11] potentially due to differences in dietary habits, GMB or host genetic background across populations. Major dietary determinants of circulating TMAO remain open for investigation in human populations.

As to the role of GMB in modulating circulating TMAO, it has been found that antibiotics can suppress blood levels of TMAO in mice[1] and humans[2]. Two recent studies using 16S rRNA gene amplicon sequencing (16S) data identified several GMB features (e.g., genera from the orders Clostridiales, Bifidobacteriales, and Bacteroidales) associated with circulating TMAO[12, 13]. However, the specific gut bacterial taxa which can metabolize the TMA-containing nutrients into TMA and thus elevate host TMAO level remain to be identified in humans. Moreover, the potential interaction between GMB and dietary factors (e.g., red meat intake) on circulating TMAO levels has been unexplored in human populations.

In this study of US Hispanics/Latinos, we aimed to: (i) confirm the association between serum TMAO and CVD; (ii) examine the associations of dietary factors with serum levels of TMAO and precursor metabolites (choline, carnitine, and betaine), and (iii) identify GMB features associated with serum TMAO.

\section{Methods}

\section{Study design and population}

The Hispanic Community Health Study/Study of Latinos (HCHS/SOL) is a prospective community-based cohort study of 16,415 Hispanic/Latino adults aged 18-74 years at recruitment who were living in four US urban areas (Bronx, NY; Chicago, IL; Miami, FL; and San Diego, CA). A two-stage area probability sample design was used to recruit participants, which has been previously described[14, 15]. A comprehensive set of interviews and a clinical examination with fasting blood draw were conducted by trained and certified staff at in-person clinic visits from 2008-2011. The HCHS/SOL Gut Origins of Latino Diabetes (GOLD) ancillary study was conducted during 2016-2018 to investigate the role of GMB composition on the risk of diabetes and other health outcomes, with a total of 3057 participants enrolled from the HCHS/SOL approximately concurrent with the second in-person visit period from 2014 to 2017[16]. The study was approved by the institutional review boards of corresponding site institutions. Written informed consent was obtained from all participants. 


\section{Ascertainment of cardiometabolic diseases}

Cardiometabolic diseases were ascertained using information collected at the 2008-2011 examination. Diabetes was defined as either current use of antidiabetic medications, or fasting glucose $\geq 126 \mathrm{mg} / \mathrm{dl}$, 2h oral glucose tolerance test plasma glucose $\geq 200 \mathrm{mg} / \mathrm{dl}$, or hemoglobin A1c $\geq 6.5 \%[17]$. Hypertension was defined as systolic or diastolic blood pressure of $\geq 140 / 90 \mathrm{mmHg}$ or currently taking antihypertensive medications. Dyslipidemia was defined as serum low-density lipoprotein cholesterol $\geq 160 \mathrm{mg} / \mathrm{dl}$, high-density lipoprotein cholesterol $<40 \mathrm{mg} / \mathrm{dl}$, or triglycerides $\geq 200 \mathrm{mg} / \mathrm{dl}$ or currently taking antihyperlipidemic medications[18]. CVD was ascertained according to self-reported physician diagnosis of coronary heart disease (CHD) (including a history of a cardiac event or procedure, or electrocardiographic evidence of myocardial infarction observed) or cerebrovascular disease or carotid revascularization (CDCR) (history of stroke, mini-stroke or transient ischemic attack, or balloon angioplasty or surgery to the arteries in the neck)[18].

\section{Assessment of diet and other covariates}

Usual diet intake was estimated from two $24-\mathrm{h}$ dietary recalls described previously[19, 20]. The first recall was collected by in-person interviews during the 2008-2011 examination, and the second recall was collected via telephone approximately 30 days after the first interview. Participants estimated portion sizes with the use of food models (in-person) or a food-amount booklet (for telephone interviews). Foods and nutrients were analyzed using the multiple-pass methods of the Nutrition Data System for Research software (version 11) from the Nutrition Coordinating Center at the University of Minnesota[21]. The present analyses mainly focus on 15 major food groups, and 36 major macro and micro nutrients were also examined in secondary analysis. Behavioral and sociodemographic characteristics of participants, including Hispanic/Latino background, smoking and alcohol consumption, education attainment, annual household income, and medical and family histories were collected using structured questionnaires[14, 15]. Physical activity was measured using the Global Physical Activity Questionnaire and the metabolic equivalent-hours/day was derived[22]. Body mass index (BMI) was computed as measured weight (in kilograms) divided by measured height (in meters) squared.

\section{Serum metabolite measurement}

Metabolomic profiling was performed on serum specimens collected during the 2008-2011 examination from 3972 participants randomly selected from the entire study population, using an untargeted liquid chromatography-mass spectrometry (LC-MS) approach based on the discoveryHD4 platform at Metabolon Inc. (Durham, NC). Details on sample extraction, separation and MS analysis have been described elsewhere[23]. Briefly, this approach utilized a Waters ACQUITY UPLC and a Thermo Scientific Q-Exactive high resolution/accurate MS interfaced with a heated electrospray ionization (HESI-II) source and Orbitrap mass analyzer operated at 35,000 mass resolution. Metabolite peaks were identified and confirmed using authentic reference standards. Metabolites were quantified using area-under-the-curve of the peaks. TMAO and three precursor metabolites (choline, carnitine, and betaine) were captured by this method and were included in the present analysis. The undetectable rates of these four metabolites were 
all less than $0.1 \%$. Missing values of the four metabolites were imputed with half of the detectable minimum values. A rank-based inverse normal transformation was applied to the metabolite data before analyses[24].

\section{Gut Microbiome Shotgun Metagenomics Sequencing}

In the GOLD ancillary study, enrolled participants were provided with a stool collection kit. For each participant, a single fecal specimen was self-collected using a disposable paper inverted hat (Protocult collection device, ABC Medical Enterprises, Inc., Rochester, MN). Detailed sample processing and DNA extraction procedures have been described previously[16]. Shotgun Metagenomics Sequencing was performed by a novel shallow-coverage method of shotgun sequencing-based Illumina NovaSeq platforms[25], boosted using inference from reference genomes of likely genomic content. The adapters and barcode indices are processed following the iTru adapter protocol[26]. Shallow shotgun data was left trimmed to remove low-quality bases that had a PHRED quality score of 25 or less using prinseq-lite $0.20 .4[27]$. The quality controlled paired-end data was then concatenated and aligned against the NCBI RefSeq representative prokaryotic genome collection (release 82[28]) using default SHOGUN [29] settings. Samples with a coverage depth less than 100,000 reads per sample were excluded. Of 3035 samples with sequencing data, 2764 samples passed all QC metrics and were included in downstream analyses. Bowtie2[30] was used to build the SHOGUN assemblies. The reads that mapped to a single reference genome were labeled with the NCBI taxonomic annotation at species level. Those reads that mapped to multiple reference genomes were labeled as the last common ancestor (LCA) of each label according to the NCBI taxonomy[29]. Cumulative sum scaling normalization was conducted for the species-level abundance of taxonomic units[31] before analyses.

\section{Statistical analysis}

We examined cross-sectional associations of TMAO and its precursor metabolites with prevalence of CVD, and its constituent components CHD and CDCR, in 3827 participants without cancer. For the analysis on dietary factors and serum levels of TMAO and its precursor metabolites, we further excluded participants with prevalent CVD $(n=279)$, with missing dietary information $(n=28)$, or with extreme dietary energy intake $(>6000 /<600 \mathrm{Kcal} /$ day in men, or $>4000 /<400 \mathrm{Kcal} /$ day in women) $(\mathrm{n}=54)$, leaving 3466 participants (Supplementary Figure 1). Next, in a subgroup of participants $(n=626)$ who had both metabolite and GMB data, we examined the associations of gut microbial alpha diversity and species abundance with TMAO levels (Supplementary Figure 1).

Characteristics of the study participants were presented according to quartile of the TMAO as means \pm standard deviations (SDs) or percentages where appropriate. Participant characteristics were compared across quartile of TMAO by using one-way ANOVA for continuous variables and Chi-square test for categorical variables. Associations of TMAO and its precursor metabolites with the prevalence of CVD, $\mathrm{CHD}$ and $\mathrm{CDCR}$ were assessed using multivariable logistic regression models with adjustment of age, sex, field center, Hispanic/Latino background, education, family income, smoking, alcohol consumption, dietary energy, physical activity, diabetes, hypertension, and dyslipidemia. Associations of dietary factors 
(including food groups and nutrients) with TMAO and its precursor metabolites were assessed using linear regression models with controlling for the aforementioned covariates. To reduce potential influences of extreme values, quintiles were created for all dietary intake variables, and the quintile ranks were used in the analyses.

The gut microbial alpha diversity and species abundance were examined for associations with serum TMAO using linear regression, after controlling for the aforementioned covariates in addition to use of antibiotics and probiotics. Next, we explored potential effect modification on diet-metabolite associations by gut microbial species. We examined associations between food groups (i.e., fish, red meat, eggs) and TMAO levels stratified by abundance of microbial species (i.e., above versus below median) that were significantly and positively associated with TMAO. We also calculated a GMB score (ranges from 0-4) based on the abundance of the four TMAO-positively-associated microbial species (<median value: 0 , and $\geq$ median value: 1 ) and examined associations of food groups with TMAO across the three levels of the GMB score (0, low GMB score; 1-2, medium GMB score; and 3-4, high GMB score). The interactions between food groups and GMB score on serum TMAO levels were tested by including the respective interaction terms in the models (e.g., red meat intake $\times$ GMB score). To examine the joint effect of red meat intake and the GMB score on serum TMAO, red meat intake was further categorized as tertiles instead of quintiles to ensure adequate sample sizes in each of 9 subgroups (red meat intake tertiles $\times 3$ GMB score categories). The Benjamini-Hochberg false discovery rate (FDR) method was used for multiple testing correction. All analyses were performed using R version 3.6.0 (https://www.r-project.org/).

\section{Results}

\section{Participant characteristics}

Characteristics of participants according to quartile of serum TMAO are presented in Table 1. Participants with a higher level of TMAO were older and less likely to be women, were more likely to be current smokers, had higher BMI and higher dietary energy intake on average, and were more likely to have diabetes, hypertension, and dyslipidemia, compared to those with a lower level of TMAO. 
Table 1

Characteristics of participants according to quartiles of serum TMAO level

Quartiles of TMAO

$P$

$$
\text { Q1 ( }=957) \quad \text { Q2 ( }=957) \quad \text { Q3 }(n=957) \quad \text { Q4 }(n=956)
$$

$\begin{array}{lllll}\text { Age, years } & 41.3( \pm 13.9) & 45.0( \pm 13.2) & 47.2( \pm 13.6) & 48.4( \pm 13.5)\end{array}$

Female, \%

$592(61.9 \%) \quad 557(58.2 \%) \quad 539(56.3 \%)$

$476(49.8 \%) \quad<$

0.001

\section{Education, \%}

$\begin{array}{llllll}1-10 \text { years } & 319(33.4 \%) & 335(35.1 \%) & 348(36.4 \%) & 363(38.0 \%) & 0.56 \\ 11-12 \text { years } & 256(26.8 \%) & 253(26.5 \%) & 249(26.0 \%) & 241(25.2 \%) & \\ \text { More than 13 years } & 380(39.8 \%) & 367(38.4 \%) & 359(37.6 \%) & 351(36.8 \%) & \end{array}$

\section{Household yearly income, \%}

\begin{tabular}{llllll} 
\$20000 or less & $577(60.3 \%)$ & $607(63.4 \%)$ & $619(64.7 \%)$ & $610(63.8 \%)$ & 0.37 \\
\hline $20001-\$ 50000$ & $323(33.8 \%)$ & $292(30.5 \%)$ & $278(29.0 \%)$ & $297(31.1 \%)$ & \\
More than \$50000 & $57(6.0 \%)$ & $58(6.1 \%)$ & $60(6.3 \%)$ & $49(5.1 \%)$
\end{tabular}

\section{Background, \%}

$\begin{array}{llllll}\text { Dominican } & 122(12.8 \%) & 96(10.1 \%) & 98(10.3 \%) & 63(6.6 \%) & 0.004 \\ \text { Central American } & 84(8.8 \%) & 91(9.5 \%) & 104(10.9 \%) & 109(11.4 \%) & \\ \text { Cuban } & 147(15.4 \%) & 159(16.7 \%) & 178(18.6 \%) & 156(16.3 \%) & \\ \text { Mexican } & 359(37.7 \%) & 332(34.8 \%) & 329(34.4 \%) & 365(38.2 \%) & \\ \text { Puerto Rican } & 148(15.5 \%) & 190(19.9 \%) & 157(16.4 \%) & 180(18.8 \%) \\ \text { South American } & 61(6.4 \%) & 52(5.5 \%) & 58(6.1 \%) & 57(6.0 \%) \\ \text { Other/more than one } & 32(3.4 \%) & 34(3.6 \%) & 32(3.3 \%) & 25(2.6 \%) \\ \text { Smoking, \% } & & & & & < \\ \text { Never } & 615(64.3 \%) & 584(61.0 \%) & 534(55.8 \%) & 507(53.1 \%) & 0.001\end{array}$

Data are mean ( \pm standard deviation) or $\mathrm{N}(\%)$.

$P$ values were calculated from the one-way ANOVA for continuous variables and Chi-square test for categorical variables.

TMAO, trimethylamine-N-oxide; BMI, body mass index; and MET, metabolic equivalent task. 


\begin{tabular}{|c|c|c|c|c|c|}
\hline & \multicolumn{4}{|c|}{ Quartiles of TMAO } & \multirow[t]{2}{*}{$P$} \\
\hline & Q1 (n= 957) & Q2 ( $n=957)$ & Q3 (n = 957) & Q4 (n= 956) & \\
\hline Former & $161(16.8 \%)$ & $182(19.0 \%)$ & $196(20.5 \%)$ & $213(22.3 \%)$ & \\
\hline Current & $180(18.8 \%)$ & $191(20.0 \%)$ & $227(23.7 \%)$ & $234(24.5 \%)$ & \\
\hline $\begin{array}{l}\text { Alcohol consumption, } \\
\text { g/day }\end{array}$ & $3.3( \pm 16.0)$ & $2.7( \pm 12.1)$ & $3.7( \pm 15.7)$ & $4.2( \pm 16.0)$ & 0.09 \\
\hline $\mathrm{BMl}, \mathrm{kg} / \mathrm{m}^{2}$ & $29.0( \pm 6.1)$ & $29.7( \pm 6.1)$ & $30.2( \pm 6.1)$ & $30.1( \pm 5.8)$ & $\stackrel{<}{<.001}$ \\
\hline $\begin{array}{l}\text { Physical activity, MET- } \\
\text { min/day }\end{array}$ & $\begin{array}{l}638.9( \pm \\
935.2)\end{array}$ & $\begin{array}{l}625.9( \pm \\
974.2)\end{array}$ & $\begin{array}{l}625.4( \pm \\
954.6)\end{array}$ & $\begin{array}{l}659.8( \pm \\
1059.2)\end{array}$ & 0.66 \\
\hline Dietary energy, kcal/day & $\begin{array}{l}1978.5( \pm \\
993.3)\end{array}$ & $\begin{array}{l}1977.0( \pm \\
966.5)\end{array}$ & $\begin{array}{l}2023.2( \pm \\
1030.1)\end{array}$ & $\begin{array}{l}2069.2( \pm \\
1119.1)\end{array}$ & 0.03 \\
\hline Diabetes, \% & $138(14.4 \%)$ & $141(14.7 \%)$ & $201(21.0 \%)$ & $267(27.9 \%)$ & $\stackrel{<}{0.001}$ \\
\hline Hypertension, \% & $201(21.0 \%)$ & $239(25.0 \%)$ & 301 (31.5\%) & $333(34.8 \%)$ & <. 0.001 \\
\hline Dyslipidemia, \% & $343(35.8 \%)$ & $365(38.2 \%)$ & $377(39.4 \%)$ & $418(43.8 \%)$ & 0.004 \\
\hline \multicolumn{6}{|c|}{ Data are mean ( \pm standard deviation) or $\mathrm{N}(\%)$. } \\
\hline \multicolumn{6}{|c|}{$\begin{array}{l}P \text { values were calculated from the one-way ANOVA for continuous variables and Chi-square test for } \\
\text { categorical variables. }\end{array}$} \\
\hline
\end{tabular}

\section{Tmao Metabolites And Cvd}

As expected, the four metabolites showed a modest-to-moderate correlation with each other except for no correlation between choline and carnitine (Supplementary Fig. 2). Serum TMAO was associated with elevated odds of CVD (odds ratio [OR] per SD increment: 1.28 [95\% confidence interval (CI): 1.12-1.48]), CHD (1.25 [95\% Cl: 1.07-1.47]), and CDCR (1.32 [95\% Cl: 1.04-1.66]) (Table 2). In addition, serum betaine, but not choline or carnitine, was also associated with elevated odds of CVD, CHD, and CDCR (Table 2). 
Table 2

Associations of serum TMAO and its precursor metabolites with prevalent cardiovascular disease

TMAO

Choline

Carnitine

Betaine

CVD $(n=279)$

\begin{tabular}{|c|c|c|c|c|}
\hline Q1 & 1.00 & 1.00 & 1.00 & 1.00 \\
\hline Q2 & $0.9(0.57-1.4)$ & $0.97(0.65-1.46)$ & $0.9(0.61-1.33)$ & $1.52(1.04-2.24)$ \\
\hline Q3 & $1.38(0.92-2.09)$ & $1.05(0.71-1.56)$ & $0.95(0.65-1.4)$ & $1.50(1.01-2.25)$ \\
\hline Q4 & $1.65(1.11-2.47)$ & $0.97(0.66-1.44)$ & $1.03(0.7-1.5)$ & $1.83(1.24-2.72)$ \\
\hline$P$ for trend & 0.001 & 0.96 & 0.79 & 0.005 \\
\hline Per 1-SD Increment & $1.28(1.12-1.48)$ & $1.06(0.93-1.22)$ & $1.03(0.9-1.17)$ & $1.19(1.04-1.36)$ \\
\hline \multicolumn{5}{|l|}{$\mathrm{CHD}(n=212)$} \\
\hline Q1 & 1.00 & 1.00 & 1.00 & 1.00 \\
\hline Q2 & $0.93(0.56-1.55)$ & $1.02(0.65-1.62)$ & $0.74(0.48-1.13)$ & $1.42(0.92-2.2)$ \\
\hline Q3 & $1.3(0.83-2.1)$ & $1.01(0.65-1.58)$ & $0.76(0.5-1.17)$ & $1.47(0.95-2.31)$ \\
\hline Q4 & $1.6(1.03-2.53)$ & $0.92(0.59-1.44)$ & $0.76(0.5-1.15)$ & $1.62(1.05-2.52)$ \\
\hline$P$ for trend & 0.009 & 0.67 & 0.26 & 0.039 \\
\hline Per 1-SD Increment & $1.25(1.07-1.47)$ & $1.04(0.9-1.21)$ & $0.93(0.81-1.08)$ & $1.14(0.98-1.33)$ \\
\hline \multicolumn{5}{|l|}{ CDCR $(n=97)$} \\
\hline Q1 & 1.00 & 1.00 & 1.00 & 1.00 \\
\hline Q2 & $1.06(0.49-2.34)$ & $1.04(0.52-2.13)$ & $1.65(0.88-3.2)$ & $1.74(0.93-3.35)$ \\
\hline Q3 & $1.76(0.9-3.67)$ & $1.34(0.7-2.67)$ & $1.28(0.66-2.53)$ & $1.62(0.82-3.22)$ \\
\hline Q4 & $1.95(1-4.05)$ & $1.32(0.69-2.6)$ & $1.73(0.92-3.35)$ & $2.33(1.24-4.49)$ \\
\hline$P$ for trend & 0.019 & 0.30 & 0.19 & 0.017 \\
\hline Per 1-SD Increment & $1.32(1.04-1.66)$ & $1.18(0.95-1.46)$ & $1.15(0.93-1.43)$ & $1.29(1.03-1.6)$ \\
\hline
\end{tabular}

Data are ORs (95\% Cls) from logistic regression models after the adjustment for age, sex, body mass index, study center, Hispanic/Latino background, physical activity, alcohol consumption, smoking, education, yearly household income, dietary energy, diabetes, hypertension, and dyslipidemia.

TMAO, trimethylamine-N-oxide; CVD, cardiovascular disease; CHD, coronary heart disease; CDCR, cerebrovascular disease or carotid revascularization. OR, odds ratio; $\mathrm{Cl}$, confidence interval; and SD, standard deviation. 


\section{Diet And Tmao Metabolites}

We next assessed associations of dietary factors with serum levels of TMAO and its precursor metabolites. After multivariable adjustment for socioeconomic and lifestyle factors, higher intakes of red meat $\left(P=6.74 \cdot 10^{-21}\right)$, fish $\left(P=7.24 \cdot 10^{-17}\right)$, and eggs $\left(P=5.11 \cdot 10^{-4}\right)$ were strongly associated with a higher level of TMAO, while intake of poultry $\left(P=5.78 \cdot 10^{-8}\right)$, dairy $\left(P=5.1 \cdot 10^{-5}\right)$, and processed meat $(P$ $=0.001$ ) were inversely associated with serum TMAO (Fig. 1A and Supplementary Table 1). In addition, egg and refined grain intakes were positively associated with serum choline $\left(P=1.37 \cdot 10^{-7}\right.$ and 0.002 , respectively) and betaine ( $P=6.59 \cdot 10^{-6}$ and 0.003 , respectively). Red meat intake was positively associated with serum carnitine $\left(P=1.84 \cdot 10^{-4}\right)$.

We then included all 15 food groups in one model (mutual adjustment) to assess independent relationships of food groups with TMAO and its precursor metabolites. Red meat, fish, and egg intakes remained strongly and positively associated with serum TMAO levels. As shown in Fig. 1B, the ascending trends of TMAO were significant as intakes of fish $\left(P=1.26 \cdot 10^{-17}\right)$, red meat $\left(P=3.33 \cdot 10^{-16}\right)$, and eggs $\left(P=3.89 \cdot 10^{-5}\right)$ increased. In addition, red meat intake was positively associated with serum carnitine $(P$ $\left.=2.17 \cdot 10^{-4}\right)$, and egg intake was positively associated with serum choline $\left(P=2.26 \cdot 10^{-7}\right)$ and betaine $(P$ $=8.66 \cdot 10^{-6}$ ), while fish intake was not associated with any of these TMAO precursors (All $P>0.05$ ).

Results for the dietary nutrients in relation to metabolites were generally in agreement with those for food groups (Supplementary Table 1). Dietary intakes of total protein, animal protein, thiamin, vitamin B12, calcium, zinc, and selenium, which were largely from animal source foods, were strongly associated with a higher level of TMAO after the multivariable adjustment (All $P \leq 0.011$ ). In addition, dietary eicosapentaenoic acid (EPA) and docosahexaenoic acid (DHA), which were mainly from fish intake, were positively associated with serum TMAO (Both $P \leq 0.003$ ). As expected, dietary choline was positively correlated with serum levels of TMAO $(P<0.001)$ and choline $(P<0.001)$.

\section{Gmb And Tmao}

We firstly assessed the relationship between three different measures of microbiome alpha diversity (i.e., Chao1, Shannon, and Simpson) and serum levels of TMAO, and did not find significant associations (Supplementary Table 2).

Among the 339 identified gut microbial species (average reads $\geq 10$ and prevalence $\geq 15 \%$ ), 9 were significantly associated with serum TMAO levels after adjusting for socioeconomic, lifestyle, antibiotic and probiotic use (FDR-adjusted $P<0.05$, Fig. 2 and Supplementary Table 3). Four of the 9 species, belonging to order Clostridiales (i.e., Oscillibacter sp. ER4, Oscillibacter sp. 1-3, Intestinimonas butyriciproducens, and Pseudoflavonifractor capillosus), were positively associated with serum TMAO, while the other 5 species (i.e., Megasphaera micronuciformis, Streptococcus mitis, Alloscardovia omnicolens, Bifidobacterium saguini, and Haemophilus influenzae) were inversely associated with serum TMAO. We did not find significant associations of these 9 species with fish, red meat, or egg intake. 
We then focused on 4 species which were positively associated with TMAO and might have the potential to produce TMAO, hypothesizing that they may modify the associations of TMAO with red meat and egg intake. As shown in Fig. 3A, there was a significant interaction between red meat intake and the GMB score based on these 4 species on serum TMAO $\left(P_{\text {interaction }}=0.013\right)$, with a stronger association between red meat intake and serum TMAO among participants with a higher GMB score compared to those with a lower score. Higher red meat intake was significantly associated with higher serum TMAO only in participants with a high GMB score, but not in those with a low or medium score (Fig. 3B). No such microbial modification was observed on the associations of fish or egg intake with TMAO (Fig. 3A). Similar results were observed when we analyzed each of these 4 species individually (Supplementary Fig. 3). Higher red meat intake was significantly associated with higher serum TMAO only in participants with higher abundance of these species (above median).

\section{Discussion}

In a population-based representative sample of US Hispanic/Latino adults, we confirmed that TMAO, a diet-derived, gut microbial related metabolite, was associated with a higher prevalence of CVD. Since 2011, it has been reported that heightened circulating TMAO was associated with an increased risk of major adverse cardiovascular events (including myocardial infarction, stroke, or death) $[1,2,5]$. The relationship between TMAO and CVD was examined in subsequent studies of different populations and confirmed in a meta-analysis of 19 cohorts which mainly included Caucasian and black participants[3]. To the best of our knowledge, this is the first study to report a positive association between TMAO and prevalent CVD in US Hispanic/Latino adults of diverse backgrounds, though further studies with prospective data in this population are needed.

Our analyses demonstrated both GMB-dependent (e.g., red meat) and GMB-independent diet (e.g., fish) sources of circulating TMAO in human populations. GMB-dependent TMAO, meaning the fraction that is produced by gut bacteria metabolism, was mainly from dietary choline and carnitine, both of which are abundant in eggs, yolks, liver, and a variety of meat $[9,32,33]$. However, TMAO is also naturally existing in seafood in a preformed state[34,35]. A recent randomized controlled trial confirmed that eggs had the highest content of choline, beef had the highest level of carnitine, while fish had 650 times more TMAO compared to eggs and beef[6]. Along with another dietary intervention study, these reports found that circulating TMAO increased over a short time after consumption of fish[6,36]. A few observational studies conducted in general populations have examined the associations of food groups with TMAO and yielded various results[8-11,37]. For example, fish and red meat intakes were positively correlated with circulating TMAO in studies from Germany and Italy[10, 11], while another study from Germany found that consumption of dairy, but not meat, eggs, or fish was positively associated with plasma TMAO[8]. In addition, a study from China found that consumption of fish but not red meat was associated with elevated urinary TMAO[9]. Our current study found that consumption of fish, red meat, and eggs were three major dietary determinants of serum TMAO in US Hispanics/Latinos; and red meat and egg intake, but not fish intake, were positively associated with serum levels of TMAO precursors. Our findings provide strong supports for two suggested major pathways of TMAO in human circulation[6, 36]. 
Previous studies in mice and humans have indicated that circulating TMAO from choline or carnitine was GMB dependent $[1,5]$. However, the specific taxa which might be associated with TMAO production have not been fully understood. Recently, several studies have explored the relationship between gut bacterial taxa and circulating levels of TMAO in humans $[5,12,13,38,39]$. For example, three intervention studies including 20 to 60 participants found that several genera (e.g., Clostridium clusters XIVa) belonging Clostridiales order were associated with elevated TMAO $[5,38,39]$. Strains from Clostridium XIVa have been confirmed to possess a choline TMA-lyase (cutC)[40]. Another two recent studies in general populations also found that genera belonging to the Clostridiales, Bacteroidales, or Desulfovibrionales order were positively associated with circulating $\operatorname{TMAO}[12,13]$. Partially consistent with previous results, the four gut microbial species which were positively associated with serum TMAO in our study all belong to the Clostridiales order. More specifically, two microbial species (i.e., Oscillibacter sp. ER4 and Oscillibacter sp. 1-3) identified in our study, belong to Oscillibacter, a genus which was previously reported to be associated with circulating TMAO levels[39, 41] and cerebrovascular disease[42]. Moreover, our bacterial gene alignment analysis indicated that three of these four gut microbial species (Oscillibacter sp. 1-3, Pseudoflavonifractor capillosus, and Intestinimonas butyriciproducens) possess homologous genes encoding carnitine monooxygenase (cntA/B), an enzyme which converts carnitine to TMA/TMAO (Supplementary Table 4). This suggests that these species may have the potential to produce TMO, though further analytical and experimental studies are needed to demonstrate the TMAproducing capability of these species.

The identified gut microbial species and their potential capability to produce TMA/TMAO from carnitine/red meat were further supported by a significant microbial modification on the red meat-TMAO association observed in this study. This finding suggests that the positive association between red meat and circulating TMAO is dependent on these gut taxa which might contribute to the processing of carnitine from dietary red meatto TMA/TMAO[10,43]. As expected, we did not find such microbial modification on the association between fish intake and serum TMAO, further supporting fish as a GMBindependent diet source of circulating TMAO[6, 36]. We also did not find such microbial modification on the association between egg intake, a major dietary source of choline[10], and serum TMAO, which is line with our bacterial gene alignment results that these gut microbial species may contain carnitine monooxygenase (cntA/B), but not choline TMA-lyase (cutC) (Supplementary Table 4). Consistently, a recent dietary intervention study also suggested that higher intake of dietary red meat may increase systemic TMAO levels through microbial TMA/TMAO production from dietary carnitine, but not choline[43]. Nevertheless, since the does-response relationship between egg consumption and circulating TMAO has been demonstrated in this and previous studies[2, 44], future studies are needed to clarify the gut microbial pathway linking dietary egg/choline consumption and microbial TMA/TMAO production in humans.

This study also identified a number of gut microbial species inversely associated with serum TMAO. Among them, it is noteworthy that Bifidobacterium saguini (FDR < 0.05) and several others (i.e., $B$. longum, $B$. breve, $B$. gallinarum, and $B$. bifidum with a raw $P<0.05$ but did not pass FDR), belongs to Bifidobacterium, a genus which has been widely reported to be inversely associated with circulating 
TMAO levels in humans[12,45] and mice[46]. In support of these findings, some members of Bifidobacterium and other gut microbiota, such as the Streptococcaceae family (Streptococcus mitis inversely associated with serum TMAO in the current study belongs to this family), have been found to convert TMAO to TMA in mice and pure culture[47], although the converting rate was relatively low.

Several limitations of our study need to be acknowledged. First, follow up of incident CVD outcomes in $\mathrm{HCHS} / \mathrm{SOL}$ is still ongoing, and at present, we are unable to examine the prospective association between serum TMAO levels and risk of CVD. Second, we used an untargeted metabolomic profiling method, which did not allow us to obtain the absolute concentrations of serum metabolites, but this would not influence association results for TMAO and other metabolites with CVD, dietary factors or GMB features. Third, ascertainment of dietary intake and serum metabolites preceded sampling of GMB by $\sim 6$ years, although human GMB has been found to be notably stable over a long period.[48] However, this time lag may bias associations towards null, and we would expect even stronger associations with concurrent data. Moreover, given the utilization of shallow shotgun sequencing method, we did not analyze the microbial functional profiles in this study. Finally, the present study included US Hispanics/Latinos, who have a distinctive dietary pattern and GMB composition[16], and tri-admixed genetic backgrounds; hence, it should take caution to generalize our findings.

\section{Conclusions}

In summary, our study confirmed the cross-sectional association between TMAO and CVD, and demonstrated that fish, red meat, and egg intakes were major dietary determinants of circulating TMAO levels in US Hispanics/Latinos. We identified several gut microbial species, which may have the potential to produce TMA/TMAO, positively associated with serum TMAO. Moreover, the association between red meat intake and serum TMAO may depend on these specific gut microbial species, supporting the essential role of gut microbiota in the TMA/TMAO production from dietary carnitine. Our findings provide evidence from a human population study supporting diet-derived TMAO formation in human circulation, in a both GMB-independent and GMB-dependent TMAO manner. This in turn may have important implications for future efforts to prevent CVD related to this pathway in human populations through diet and GMB modification.

\section{Abbreviations}

TMAO

trimethylamine-N-oxide

GMB

gut microbiota

CVD

cardiovascular disease

TMA

trimethylamine

Page 14/ 24 
HCHS/SOL

Hispanic Community Health Study/Study of Latinos

GOLD

Gut Origins of Latino Diabetes

$\mathrm{CHD}$

coronary heart disease

CDCR

cerebrovascular disease or carotid revascularization

BMI

body mass index

LC-MS

liquid chromatography-mass spectrometry

LCA

last common ancestor

SD

standard deviation

FDR

false discovery rate

OR

odds ratio

$\mathrm{Cl}$

confidence interval

EPA

eicosapentaenoic acid

DHA

docosahexaenoic acid

SSB

sugar-sweetened beverage

\section{Declarations}

\section{Ethics approval and consent to participate}

The study was approved by the institutional review boards of corresponding site institutions. Names of the ethics committees and the committees' reference number were available on the HCHS/SOL website: https://sites.cscc.unc.edu/hchs/. Written informed consent was obtained from all participants.

\section{Consent of publication}

Not applicable 
All data generated or analyzed during this study are included in this published article and its supplementary information files. All other data is available from the corresponding author.

\section{Competing interests}

The authors declare that they have no competing interests.

\section{Funding}

The Hispanic Community Health Study/Study of Latinos is a collaborative study supported by contracts from the National Heart, Lung, and Blood Institute (NHLBI) to the University of North Carolina (HHSN268201300001I / N01-HC-65233), University of Miami (HHSN268201300004I / N01-HC-65234), Albert Einstein College of Medicine (HHSN268201300002I / N01-HC-65235), University of Illinois at Chicago (HHSN268201300003I / N01-HC-65236 Northwestern Univ), and San Diego State University (HHSN268201300005I / N01-HC-65237). The following Institutes/Centers/Offices have contributed to the $\mathrm{HCHS} / \mathrm{SOL}$ through a transfer of funds to the NHLBI: National Institute on Minority Health and Health Disparities (NIMHD), National Institute on Deafness and Other Communication Disorders, National Institute of Dental and Craniofacial Research, National Institute of Diabetes and Digestive and Kidney Diseases (NIDDK), National Institute of Neurological Disorders and Stroke, and NIH Institution-Office of Dietary Supplements.

This work is supported by the NIDDK R01DK119268 and NHLBI R01HL060712, and other funding sources for this study include UM1 HG008898 from the National Human Genome Research Institute, R01MD011389 from the NIMHD, and R01HL140976 from the NHLBI. Z. Mei was supported by Fudan University Exchange Program Scholarship for Doctoral Students.

\section{Authors' contributions}

QQ, ZM, and GCC conceived the study. ZM and ZW performed statistical analysis. ZM drafted the manuscript. GCC, ZW, and QQ critically revised the manuscript. RCK, RDB, QQ, and MLD collected the data and specimens from the HCHS/SOL participants and obtained funding. ZW, MU, YVB, GH, RSB did the gut microbial sequencing analysis. MU and RDB did the processing of the HCHS/SOL fecal samples. BY and EB did the metabolome profiling analysis. JL, JSWN, LH, JC, and YZ edited and reviewed the manuscript. $\mathrm{QQ}$ is the guarantor of this work and had full access to all the data in the study and takes responsibility for the integrity of the data and the accuracy of the data analysis. All authors read and approved the final manuscript.

\section{Acknowledgments}

The authors thank all the investigators, staff, and participants of HCHS/SOL for their important contributions.

\section{References}


1. Wang Z, Klipfell E, Bennett BJ, Koeth R, Levison BS, DuGar B, Feldstein AE, Britt EB, Fu X, Chung Y-M. Gut flora metabolism of phosphatidylcholine promotes cardiovascular disease. Nature. 2011;472:57.

2. Tang WW, Wang Z, Levison BS, Koeth RA, Britt EB, Fu X, Wu Y, Hazen SL. Intestinal microbial metabolism of phosphatidylcholine and cardiovascular risk. N Engl J Med. 2013;368:1575-84.

3. Heianza Y, Ma W, Manson JE, Rexrode KM, Qi L. Gut microbiota metabolites and risk of major adverse cardiovascular disease events and death: a systematic review and meta-analysis of prospective studies. Journal of the American Heart Association. 2017;6:e004947.

4. He Z, Chen Z-Y. The origin of trimethylamine-N-oxide (TMAO) and its role in development of atherosclerosis. Journal of Food Bioactives. 2018;2:28-36-28-36.

5. Koeth RA, Wang Z, Levison BS, Buffa JA, Org E, Sheehy BT, Britt EB, Fu X, Wu Y, Li L. Intestinal microbiota metabolism of L-carnitine, a nutrient in red meat, promotes atherosclerosis. Nature medicine. 2013;19:576.

6. Cho CE, Taesuwan S, Malysheva OV, Bender E, Tulchinsky NF, Yan J, Sutter JL, Caudill MA. Trimethylamine-N-oxide (TMAO) response to animal source foods varies among healthy young men and is influenced by their gut microbiota composition: a randomized controlled trial. Molecular nutrition food research. 2017;61:1600324.

7. Bjørndal B, Ramsvik M, Lindquist C, Nordrehaug J, Bruheim I, Svardal A, Nygård O, Berge R. A phospholipid-protein complex from antarctic krill reduced plasma homocysteine levels and increased plasma trimethylamine-N-oxide (TMAO) and carnitine levels in male Wistar rats. Marine drugs. 2015;13:5706-21.

8. Rohrmann S, Linseisen J, Allenspach M, von Eckardstein A, Müller D. Plasma concentrations of trimethylamine-N-oxide are directly associated with dairy food consumption and low-grade inflammation in a German adult population. J Nutr. 2015;146:283-9.

9. Yu D, Shu XO, Rivera ES, Zhang X, Cai Q, Calcutt MW, Xiang YB, Li H, Gao YT, Wang TJ. Urinary Levels of Trimethylamine-N-Oxide and Incident Coronary Heart Disease: A Prospective Investigation Among Urban Chinese Adults. Journal of the American Heart Association. 2019;8:e010606.

10. Krüger R, Merz B, Rist MJ, Ferrario PG, Bub A, Kulling SE, Watzl B. Associations of current diet with plasma and urine TMAO in the KarMeN study: Direct and indirect contributions. Molecular nutrition food research. 2017;61:1700363.

11. Barrea L, Annunziata G, Muscogiuri G, Laudisio D, Di Somma C, Maisto M, Tenore GC, Colao A, Savastano S: Trimethylamine N-oxide, Mediterranean diet, and nutrition in healthy, normal-weight adults: also a matter of sex? Nutrition 2019, 62:7-17.

12. Manor O, Zubair N, Conomos MP, Xu X, Rohwer JE, Krafft CE, Lovejoy JC, Magis AT. A multi-omic association study of trimethylamine N-oxide. Cell reports. 2018;24:935-46.

13. Fu BC, Hullar MA, Randolph TW, Franke AA, Monroe KR, Cheng I, Wilkens LR, Shepherd JA, Madeleine MM, Le Marchand L. Associations of plasma trimethylamine N-oxide, choline, carnitine, and betaine with inflammatory and cardiometabolic risk biomarkers and the fecal microbiome in the Multiethnic Cohort Adiposity Phenotype Study. The American Journal of Clinical Nutrition 2020. 
14. LaVange LM, Kalsbeek WD, Sorlie PD, Avilés-Santa LM, Kaplan RC, Barnhart J, Liu K, Giachello A, Lee DJ, Ryan J. Sample design and cohort selection in the Hispanic Community Health Study/Study of Latinos. Ann Epidemiol. 2010;20:642-9.

15. Sorlie PD, Avilés-Santa LM, Wassertheil-Smoller S, Kaplan RC, Daviglus ML, Giachello AL, Schneiderman N, Raij L, Talavera G, Allison M. Design and implementation of the Hispanic community health study/study of Latinos. Ann Epidemiol. 2010;20:629-41.

16. Kaplan RC, Wang Z, Usyk M, Sotres-Alvarez D, Daviglus ML, Schneiderman N, Talavera GA, Gellman MD, Thyagarajan B, Moon J-Y. Gut microbiome composition in the Hispanic Community Health Study/Study of Latinos is shaped by geographic relocation, environmental factors, and obesity. Genome biology. 2019;20:219.

17. Schneiderman N, Llabre M, Cowie CC, Barnhart J, Carnethon M, Gallo LC, Giachello AL, Heiss G, Kaplan RC, LaVange LM. Prevalence of diabetes among Hispanics/Latinos from diverse backgrounds: the Hispanic community health study/study of Latinos (HCHS/SOL). Diabetes Care. 2014;37:2233-9.

18. Daviglus ML, Talavera GA, Avilés-Santa ML, Allison M, Cai J, Criqui MH, Gellman M, Giachello AL, Gouskova N, Kaplan RC. Prevalence of major cardiovascular risk factors and cardiovascular diseases among Hispanic/Latino individuals of diverse backgrounds in the United States. Jama. 2012;308:1775-84.

19. Siega-Riz AM, Sotres-Alvarez D, Ayala GX, Ginsberg M, Himes JH, Liu K, Loria CM, MossavarRahmani Y, Rock CL, Rodriguez B. Food-group and nutrient-density intakes by Hispanic and Latino backgrounds in the Hispanic Community Health Study/Study of Latinos. Am J Clin Nutr. 2014;99:1487-98.

20. Wang X, Jung M, Mossavar-Rahmani Y, Sotres-Alvarez D, Espinoza Giacinto RA, Pirzada A, Reina SA, Casagrande SS, Wang T, Avilés-Santa ML. Macronutrient intake, diagnosis status, and glycemic control among US Hispanics/Latinos with diabetes. The Journal of Clinical Endocrinology Metabolism. 2016;101:1856-64.

21. Schakel SF, Buzzard IM, Gebhardt SE. Procedures for estimating nutrient values for food composition databases. Journal of food composition analysis. 1997;10:102-14.

22. Arredondo EM, Sotres-Alvarez D, Stoutenberg M, Davis SM, Crespo NC, Carnethon MR, Castañeda SF, Isasi CR, Espinoza RA, Daviglus ML. Physical activity levels in US Latino/Hispanic adults: results from the Hispanic community health study/study of Latinos. Am J Prev Med. 2016;50:500-8.

23. Zheng X, Zhao A, Xie G, Chi Y, Zhao L, Li H, Wang C, Bao Y, Jia W, Luther M, et al. Melamine-induced renal toxicity is mediated by the gut microbiota. Sci Transl Med. 2013;5:172ra122.

24. Yang J, Loos RJ, Powell JE, Medland SE, Speliotes EK, Chasman DI, Rose LM, Thorleifsson G, Steinthorsdottir V, Mägi R. FTO genotype is associated with phenotypic variability of body mass index. Nature. 2012;490:267.

25. Costello M, Fleharty M, Abreu J, Farjoun Y, Ferriera S, Holmes L, Granger B, Green L, Howd T, Mason T. Characterization and remediation of sample index swaps by non-redundant dual indexing on 
massively parallel sequencing platforms. BMC Genomics. 2018;19:332.

26. Glenn TC, Nilsen RA, Kieran TJ, Sanders JG, Bayona-Vásquez NJ, Finger JW, Pierson TW, Bentley KE, Hoffberg SL, Louha S. Adapterama I: universal stubs and primers for 384 unique dual-indexed or 147,456 combinatorially-indexed Illumina libraries (iTru \& iNext). PeerJ. 2019;7:e7755.

27. Schmieder R, Edwards R. Quality control and preprocessing of metagenomic datasets. Bioinformatics. 2011;27:863-4.

28. Al-Ghalith G, Knights D: BURST enables optimal exhaustive DNA alignment for big data. DOI: doi org/105281/zenodo 2017, 806850.

29. Hillmann B, Al-Ghalith GA, Shields-Cutler RR, Zhu Q, Gohl DM, Beckman KB, Knight R, Knights D. Evaluating the information content of shallow shotgun metagenomics. Msystems. 2018;3:e0006900018.

30. Langmead B, Salzberg SL. Fast gapped-read alignment with Bowtie 2. Nature methods. 2012;9:357.

31. Paulson JN, Stine OC, Bravo HC, Pop M. Differential abundance analysis for microbial marker-gene surveys. Nat Methods. 2013;10:1200-2.

32. Patterson KY, Bhagwat SA, Williams JR, Howe JC, Holden J, Zeisel S, Dacosta K, Mar M: USDA database for the choline content of common foods, release two. Nutrient Data Laboratory, Beltsville Human Nutrition Research Center, ARS, USDA 2008.

33. Malinowska AM, Szwengiel A, Chmurzynska A. Dietary, anthropometric, and biochemical factors influencing plasma choline, carnitine, trimethylamine, and trimethylamine-N-oxide concentrations. Int J Food Sci Nutr. 2017;68:488-95.

34. Cho CE, Caudill MA: Trimethylamine-N-oxide: friend, foe, or simply caught in the cross-fire? Trends in Endocrinology \& Metabolism 2017, 28:121-130.

35. Landfald B, Valeur J, Berstad A, Raa J. Microbial trimethylamine-N-oxide as a disease marker: something fishy? Microbial ecology in health disease. 2017;28:1327309.

36. Cheung W, Keski-Rahkonen P, Assi N, Ferrari P, Freisling H, Rinaldi S, Slimani N, Zamora-Ros R, Rundle $\mathrm{M}$, Frost G. A metabolomic study of biomarkers of meat and fish intake. Am J Clin Nutr. 2017;105:600-8.

37. Genoni A, Christophersen CT, Lo J, Coghlan M, Boyce MC, Bird AR, Lyons-Wall P, Devine A. Long-term Paleolithic diet is associated with lower resistant starch intake, different gut microbiota composition and increased serum TMAO concentrations. European journal of nutrition 2019:1-14.

38. Wu W-K, Chen C-C, Liu P-Y, Panyod S, Liao B-Y, Chen P-C, Kao H-L, Kuo H-C, Kuo C-H, Chiu TH. Identification of TMAO-producer phenotype and host-diet-gut dysbiosis by carnitine challenge test in human and germ-free mice. Gut. 2019;68:1439-49.

39. Schmedes M, Brejnrod AD, Aadland EK, Kiilerich P, Kristiansen K, Jacques H, Lavigne C, Graff IE, Eng $\varnothing$, Holthe A. The Effect of Lean-Seafood and Non-Seafood Diets on Fecal Metabolites and Gut Microbiome: Results from a Randomized Crossover Intervention Study. Molecular nutrition food research. 2019;63:1700976. 
40. Rath S, Heidrich B, Pieper DH, Vital M. Uncovering the trimethylamine-producing bacteria of the human gut microbiota. Microbiome. 2017;5:54.

41. Wang S, Xia G, He Y, Liao S, Yin J, Sheng H, Zhou H. Distribution characteristics of trimethylamine Noxide and its association with gut microbiota. Nan fang yi ke da xue xue bao = Journal of Southern Medical University. 2016;36:455-60.

42. Yin J, Liao SX, He Y, Wang S, Xia GH, Liu FT, Zhu JJ, You C, Chen Q, Zhou L. Dysbiosis of gut microbiota with reduced trimethylamine-N-oxide level in patients with large-artery atherosclerotic stroke or transient ischemic attack. Journal of the American Heart Association. 2015;4:e002699.

43. Wang Z, Bergeron N, Levison BS, Li XS, Chiu S, Jia X, Koeth RA, Li L, Wu Y, Tang WW. Impact of chronic dietary red meat, white meat, or non-meat protein on trimethylamine N-oxide metabolism and renal excretion in healthy men and women. European heart journal. 2019;40:583-94.

44. Miller CA, Corbin KD, da Costa K-A, Zhang S, Zhao X, Galanko JA, Blevins T, Bennett BJ, O'Connor A, Zeisel SH. Effect of egg ingestion on trimethylamine-N-oxide production in humans: a randomized, controlled, dose-response study. Am J Clin Nutr. 2014;100:778-86.

45. Zhang C, Yin A, Li H, Wang R, Wu G, Shen J, Zhang M, Wang L, Hou Y, Ouyang H. Dietary modulation of gut microbiota contributes to alleviation of both genetic and simple obesity in children. EBioMedicine. 2015;2:968-84.

46. Chen M-I, Yi L, Zhang Y, Zhou X, Ran L, Yang J, Zhu J-d. Zhang Q-y, Mi M-t: Resveratrol attenuates trimethylamine-N-oxide (TMAO)-induced atherosclerosis by regulating TMAO synthesis and bile acid metabolism via remodeling of the gut microbiota. MBio. 2016;7:e02210-5.

47. Hoyles L, Jiménez-Pranteda ML, Chilloux J, Brial F, Myridakis A, Aranias T, Magnan C, Gibson GR, Sanderson JD, Nicholson JK. Metabolic retroconversion of trimethylamine $\mathrm{N}$-oxide and the gut microbiota. Microbiome. 2018;6:73.

48. Faith JJ, Guruge JL, Charbonneau M, Subramanian S, Seedorf H, Goodman AL, Clemente JC, Knight $\mathrm{R}$, Heath AC, Leibel RL. The long-term stability of the human gut microbiota. Science. 2013;341:1237439.

\section{Figures}


A

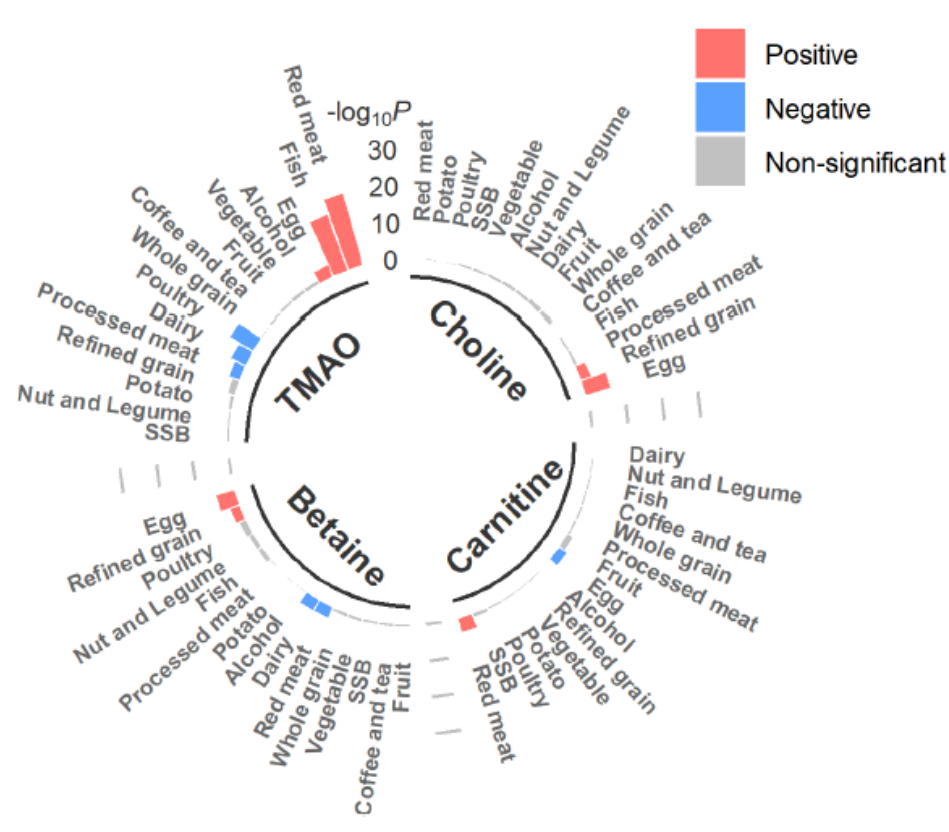

B
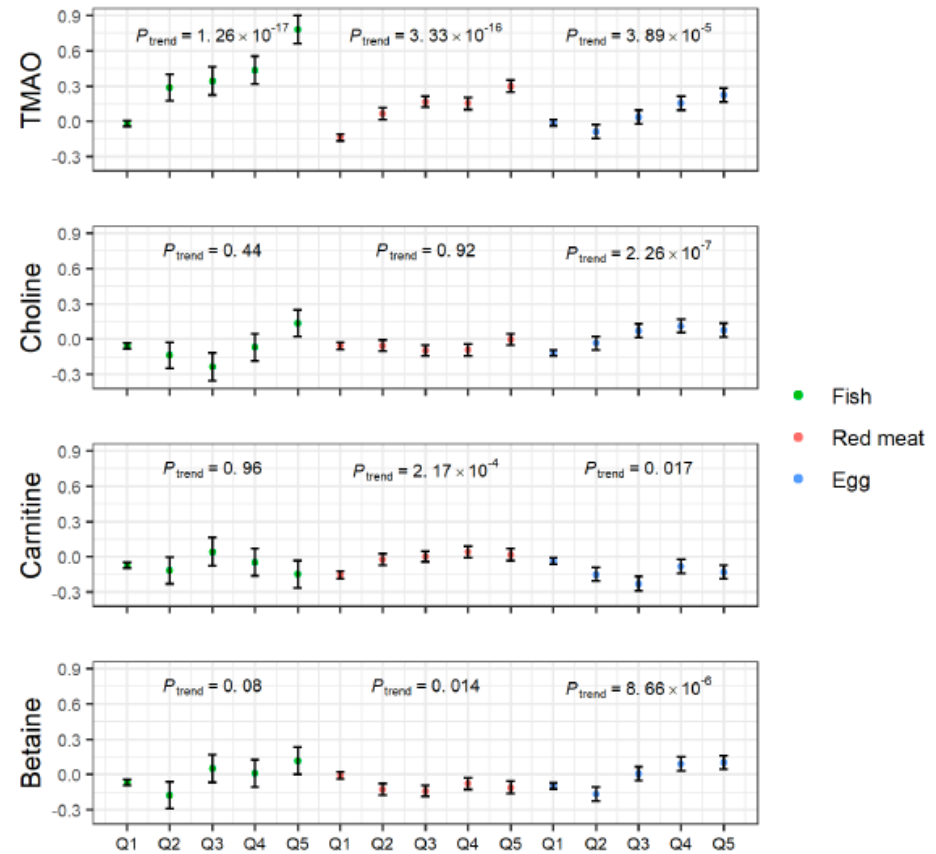

\section{Figure 1}

Associations of food groups with serum TMAO and its precursor metabolites. A. Associations (-log10P) of 15 food groups with serum TMAO and its precursor metabolites. Data are $\mathrm{P}$ values from linear regression after adjustment for age, sex, field center, Hispanic/Latino background, education, family income, smoking, alcohol consumption, dietary energy, physical activity, diabetes, hypertension, and dyslipidemia. Red bars indicate positive associations (FDR-adjusted $P<0.05$ ), blue bars indicate inverse associations (FDR-adjusted $\mathrm{P}<0.05$ ), and gray bars indicate non-significant (FDR-adjusted $\mathrm{P} \geq 0.05$ ). $\mathrm{B}$. Circulating levels of TMAO and its precursor metabolites (inverse normal transformation) across quintiles of fish, red meat, and eggs intakes. The dots and error bars are the means and standard errors estimated from linear regression models, which included all 15 food groups in the same model after multivariable adjustment for age, sex, field center, Hispanic/Latino background, education, family income, smoking, alcohol consumption, dietary energy, physical activity, diabetes, hypertension, and dyslipidemia. SSB, sugar-sweetened beverage; TMAO, trimethylamine-N-oxide; and FDR, false discovery rate. 


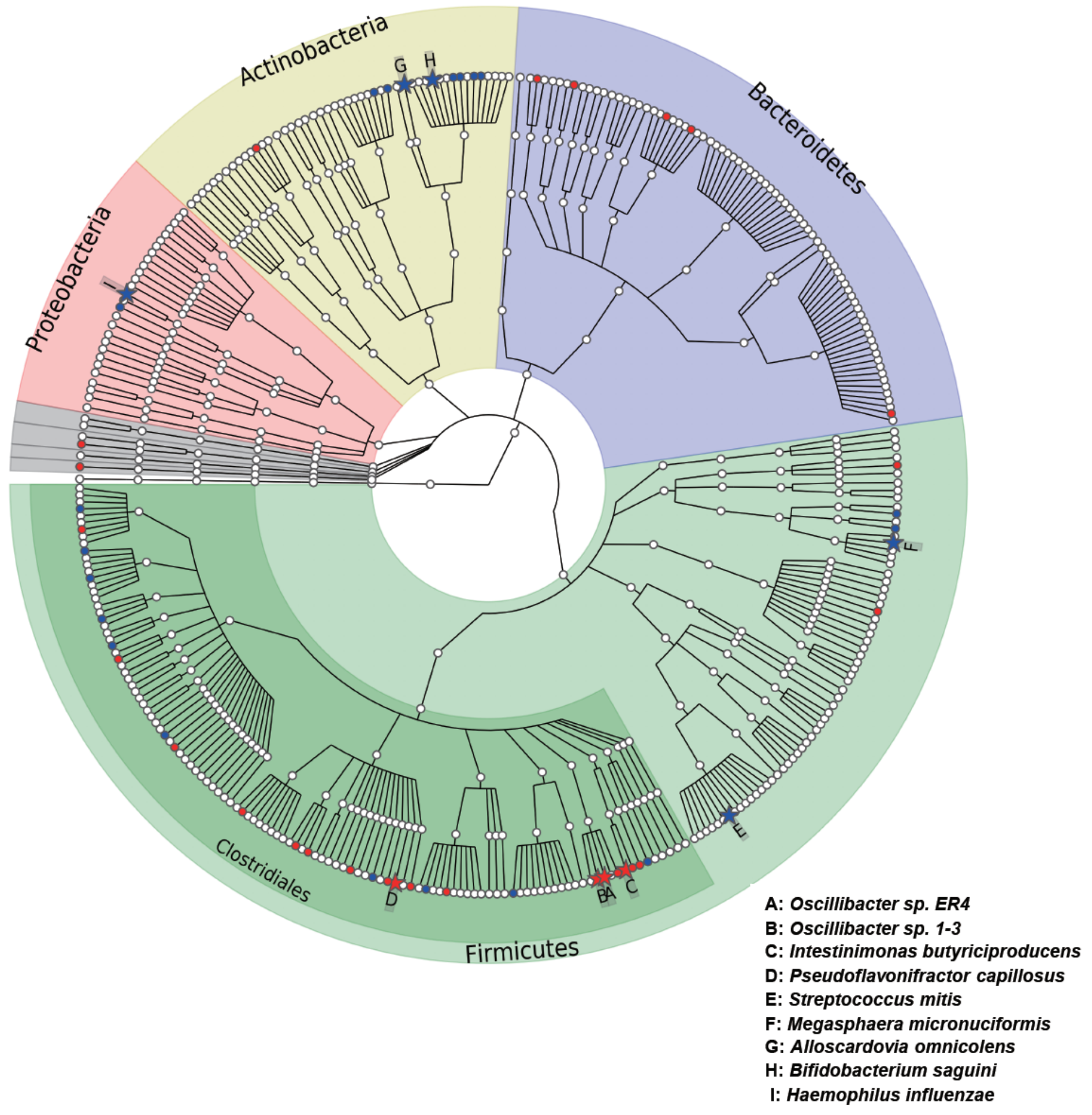

Figure 2

Phylogenetic tree of taxonomic features in association with serum TMAO levels. Species associated with TMAO at FDR-adjusted $\mathrm{P}<0.05$ were highlighted as solid stars and noted by capital letters, and species with a raw $\mathrm{P}<0.05$ were highlighted as solid circles. Solid stars or circles with red indicating positive associations while blue indicating negative associations. $P$ values were estimated from linear regression models after controlling for age, sex, body mass index, center, background, physical activity, alcohol consumption, smoking, education, yearly household income, dietary energy, and diabetes, hypertension, 
dyslipidemia, and use of antibiotics and probiotics. TMAO, trimethylamine-N-oxide; and FDR, false discovery rate.

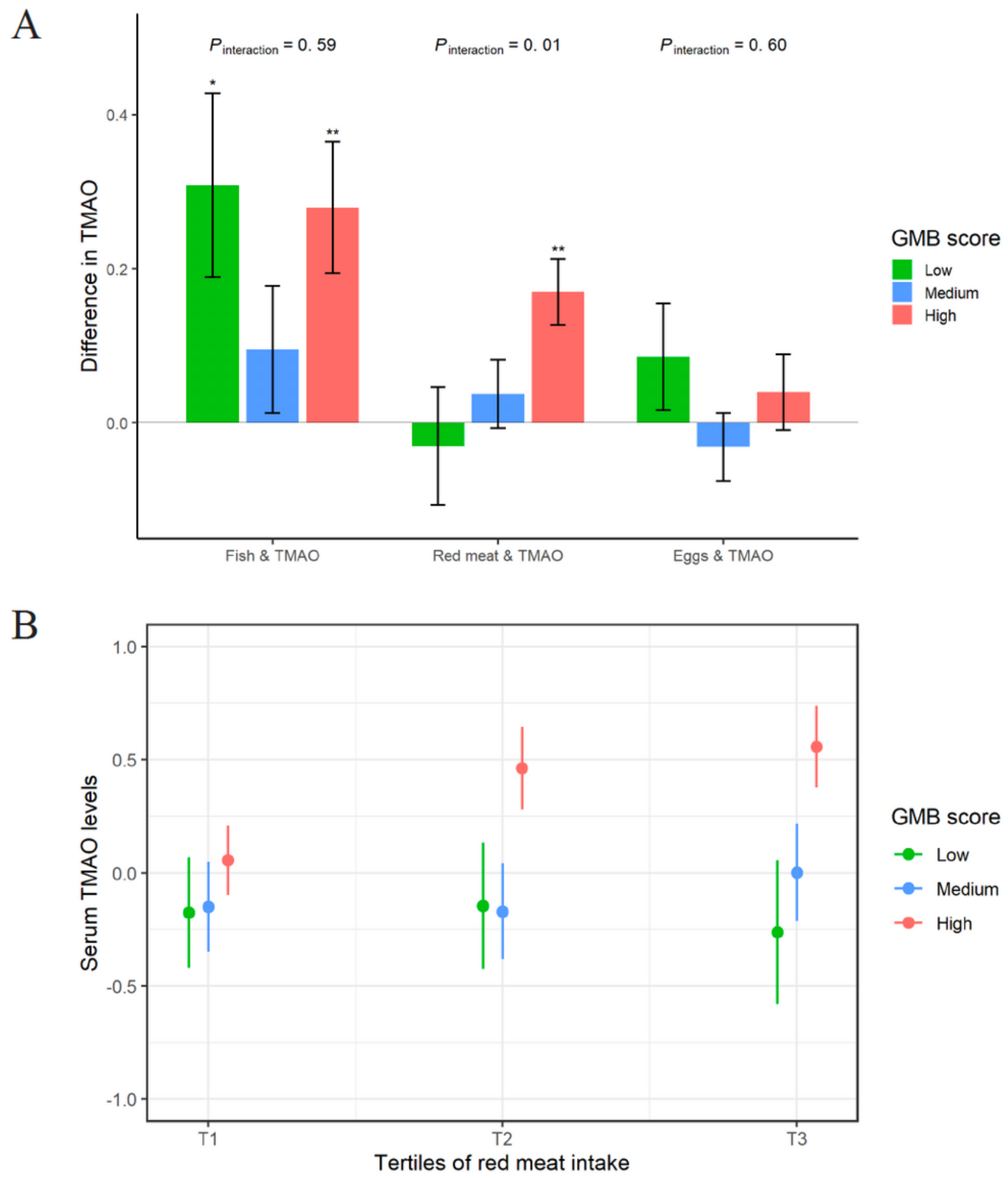

\section{Figure 3}

Associations of fish, red meat, and eggs intake with TMAO according to the GMB score. A. Data are effect size (Beta coefficients and standard errors) of food intake (per quintile) on serum TMAO levels, after adjustment for age, sex, body mass index, center, background, physical activity, alcohol consumption, 
smoking, education, yearly household income, dietary energy, and diabetes, hypertension, dyslipidemia, and use of antibiotics and probiotics. The GMB score (ranges from 0-4) was calculated based on the abundance of the four TMAO-positively-associated microbial species ( $<$ median value: 0 , and $\geq$ median value: 1): 0, low GMB score ( $n=137) ; 1-2$, medium GMB score $(n=242)$; and 3-4, high GMB score $(n=247)$. ${ }^{*} \mathrm{P}<0.05$ and ${ }^{* * P}<0.01$ were estimated from multivariable linear regression models. Pinteraction were calculated by adding a multiplicative factor in the multivariable linear regression models. B. TMAO levels (inverse normal transformation) across tertiles (instead of quintiles to ensure adequate sample sizes) of red meat intake according to GMB score. Data are means and standard errors estimated from linear regression after adjustment for age, sex, body mass index, center, background, physical activity, alcohol consumption, smoking, education, yearly household income, dietary energy, and diabetes, hypertension, and dyslipidemia. P for trend across tertiles of red meat intake were $0.83,0.40$, and $6.21 \times 10-4$ in the low, medium, and high GMB score groups, respectively. TMAO, trimethylamine-N-oxide; GMB, gut microbiota.

\section{Supplementary Files}

This is a list of supplementary files associated with this preprint. Click to download.

- Additionalfile1.docx

- Additionalfile2.xlsx 